Old Dominion University

ODU Digital Commons

Electrical \& Computer Engineering Faculty

Publications

Electrical \& Computer Engineering

2019

\title{
Carbon Multicharged Ion Generation From Laser-Spark Ion Source
}

Md. Mahmudur Rahman

Old Dominion University, mrahm003@odu.edu

Oguzhan Balki

Hani E. Elsayed-Ali

Old Dominion University, helsayed@odu.edu

Follow this and additional works at: https://digitalcommons.odu.edu/ece_fac_pubs

Part of the Electrical and Computer Engineering Commons, and the Plasma and Beam Physics

Commons

Original Publication Citation

Rahman, M. M., Balki, O., \& Elsayed-Ali, H. E. (2019). Carbon multicharged ion generation from laser-spark ion source. Review of Scientific Instruments, 90(9), 093303. doi:10.1063/1.5110402

This Article is brought to you for free and open access by the Electrical \& Computer Engineering at ODU Digital Commons. It has been accepted for inclusion in Electrical \& Computer Engineering Faculty Publications by an authorized administrator of ODU Digital Commons. For more information, please contact digitalcommons@odu.edu. 


\title{
Carbon multicharged ion generation from laser-spark ion source
}

\author{
Cite as: Rev. Sci. Instrum. 90, 093303 (2019); doi: 10.1063/1.5110402 \\ Submitted: 16 May 2019 - Accepted: 19 August 2019 • \\ Published Online: 6 September 2019
}

Md. Mahmudur Rahman, ${ }^{1,2}$ (D) Oguzhan Balki, ${ }^{\text {(D) }}$ and Hani E. Elsayed-Ali ${ }^{1,2, a)}$

\begin{abstract}
AFFILIATIONS
${ }^{1}$ Department of Electrical and Computer Engineering, Old Dominion University, Norfolk, Virginia 23529, USA

${ }^{2}$ Applied Research Center, 12050 Jefferson Avenue, Newport News, Virginia 23606, USA
\end{abstract}

a) Author to whom correspondence should be addressed: helsayed@odu.edu

\begin{abstract}
Multicharged carbon ions are generated by using a laser-assisted spark-discharge ion source. A Q-switched Nd:YAG laser pulse (1064 nm, $\left.7 \mathrm{~ns}, \leq 4.5 \times 10^{9} \mathrm{~W} / \mathrm{cm}^{2}\right)$ focused onto the surface of a glassy carbon target results in its ablation. The spark-discharge $(\sim 1.2 \mathrm{~J}$ energy, $\sim 1 \mu \mathrm{s}$ duration) is initiated along the direction of the plume propagation between the target surface and a grounded mesh that is parallel to the target surface. Ions emitted from the laser-spark plasma are detected by their time-of-flight using a Faraday cup. The ion energy-to-charge ratio is analyzed by a three-mesh retarding field analyzer. In one set of experiments, the laser plasma is generated by target ablation using a $50 \mathrm{~mJ}$ laser pulse. In another set of experiments, $\sim 1.2 \mathrm{~J}$ spark-discharge energy is coupled to the expanding plasma to increase the plasma density and temperature that results in the generation of carbon multicharged ions up to $\mathrm{C}^{6+}$. A delay-generator is used to control the time delay between the laser pulse and the thyratron trigger. Ion generation from a laser pulse when a high DC voltage is applied to the target is compared to that when a spark-discharge with an equivalent pulsed voltage is applied to the target. The laser-coupled spark-discharge ( $7 \mathrm{kV}$ peak voltage, $810 \mathrm{~A}$ peak current) increases the maximum detected ion charge state from $\mathrm{C}^{4+}$ to $\mathrm{C}^{6+}$, accompanied by an increase in the ion yield by a factor of $\sim 6$ compared to applying $7.0 \mathrm{kV}$ DC voltage to the target.
\end{abstract}

Published under license by AIP Publishing. https://doi.org/10.1063/1.5110402

\section{INTRODUCTION}

Carbon ion sources are widely used in many applications including surface hardening of iron, ${ }^{1}$ the growth of diamondlike thin films, ${ }^{2}$ carbon ion radiotherapy, ${ }^{3}$ bandgap modification of $\mathrm{TiO}_{2},{ }^{4}$ and synthesis of multilayer graphene. ${ }^{5}$ The carbon multicharged ions (MCIs) have several advantages compared to the singly charged carbon ions. MCIs require less accelerating voltage to acquire kinetic energy from an electric field compared to singly charged ions. Similarly, smaller magnetic fields can be used to focus and bend the MCIs compared to singly charged ions. ${ }^{6} \mathrm{MCIs}$ are also attractive for applications that include ion implantation, ${ }^{7}$ ion lithography, ${ }^{8}$ surface patterning and cleaning, and carbon ion cancer treatment. ${ }^{10,11}$

The use of laser plasma as an efficient source of highly charged ions was reported as early as $1969 .{ }^{12}$ Most of the initial laser ion sources were focused on elements with a low atomic number but later extended to heavier elements. ${ }^{13}$ Laser-driven ion acceleration, using a nanosecond laser pulse was demonstrated in a number of different experiments. ${ }^{14}$ The laser plasma requires no differential pumping, as in the case of electron cyclotron resonance and electron beam ion trap sources. ${ }^{15,16}$ Laser-ablation generates an intense laser-plasma from any solid target. The generation of $\mathrm{Al}^{4+}$ and $\mathrm{C}^{4+}$ by using a ns Nd:YAG laser pulse from solid targets was previously reported. ${ }^{17,18}$ An Nd:YAG laser pulse generated carbon ions which were injected into a high current radio frequency quadrupole (RFQ) linac. The $\mathrm{C}^{6+}$ ion beam with a current more than $10 \mathrm{~mA}$ was accelerated by the RFQ linac. ${ }^{19}$ The ion yield of the laser ion source depends on the laser pulse energy, pulse width, spot size, and target material. The charge separation from the laser plasma can be achieved by creating a uniform ion-accelerating external electric field. For laser ion sources, scaling ion yield is accomplished by increasing the laser pulse energy. This approach is constrained by the need for larger laser systems and a reduced pulse repetition rate. Laser-spark ion sources are attractive because only a low-energy laser pulse is required for target ablation, while the energy for plasma heating mainly comes from a spark-discharge coupled to the laser plasma. $^{20,21}$ 
Cathodic-arcs, also called a metal-vapor-vacuum-arc or simply a vacuum-arc, are also used for ion generation. ${ }^{22}$ Cathodicarcs occur at different gas pressures with the background gas significantly participating in the discharge processes. ${ }^{23}$ Cathodic-arcs have been investigated for applications in thin film deposition, ion implantation, and high current switches. ${ }^{24}$ The plasma generated by cathodic-arcs is highly ionized and contains a large number of MCIs with average kinetic energies of up to $100 \mathrm{eV} .^{25}$ The stability of the cathodic-arc depends on the arc energy, background pressure, electrode temperature, electrode shape, and electrode surface morphology. ${ }^{26}$ The arc current is localized to cathode spots that are necessary to provide sufficient power density for plasma heating, electron emission, and current transport between the cathode and the anode. ${ }^{26}$ The metal plasma that is generated at the cathode spot contains an ion drift energy of about $10-200 \mathrm{eV}$ depending on the ion mass.

Coupling the arc to laser plasma is used as an effective deposition method. Deposition of diamondlike carbon (DLC) films by the laser-arc method was reported with a deposition rate of $5 \mathrm{~nm} / \mathrm{s}^{28}$ Laser-arc deposition was also used in industrial hard coating applications where different substrate materials were coated by DLC resulting in films with frictional coefficient $\sim 0.1$ and hardness up to $80 \mathrm{GPa}$ compared to steel. ${ }^{29}$ Extreme ultraviolet light was generated by hybrid laser-assisted-vacuum-arc discharge plasmas using Sn-coated rotating-disk-electrodes. ${ }^{30}$ A laser ablation-assisted plasma discharge source was used to generate $\mathrm{Al}$ ions, where laser ablation was accomplished by focusing a $\mathrm{KrF}$ excimer laser (1.2 J pulse energy, $40 \mathrm{~ns}$ pulse width, $248 \mathrm{~nm}$ wavelength) onto a solid Al target with a fluence of approximately $10 \mathrm{~J} / \mathrm{cm}^{2}{ }^{31}$ Henig et al. generated $C^{6+}$ ions from a nanometer-thick DLC foil by using linearly and circularly polarized laser pulses. A peak $\mathrm{C}^{6+}$ ion energy distribution centered at $30 \mathrm{MeV}$ was demonstrated for a circularly polarized $0.7 \mathrm{~J}$ laser pulse interacting with a $5.3 \mathrm{~nm}$ thick foil. ${ }^{32}$ Takagi et al. reported the generation of $\mathrm{Fe}^{16+}$ with ion energies from several hundreds of $\mathrm{eV}$ to several tens $\mathrm{keV}$ using a $125 \mathrm{~J}$ spark-discharge pulse energy coupled to a laser pulse. ${ }^{33}$ Shaim et al. reported an $\mathrm{Nd}$ :YAG laser-spark ion source where the total $\mathrm{Al}$ ion yield from the laser plasma was enhanced by a factor of $\sim 9$ and the maximum charge state was increased from $\mathrm{Al}^{4+}$ to $\mathrm{Al}^{6+}$ with spark-discharge coupling. In that case, the spark-discharge was generated by a $0.1 \mu \mathrm{F}$ capacitor charged to a maximum of $5.0 \mathrm{kV}$ and the discharge time was $\sim 1.2 \mu \mathrm{s} .{ }^{20}$ Rahman et al. reported on the generation of $\mathrm{Al}^{8+}$ by using a thyratron-triggered high-voltage pulse generator with a pulse forming network (PFN). ${ }^{34}$ Balki et al. reported the generation of $\mathrm{C}^{6+}$ and enhancement in the total ion charge by a factor of $\sim 6$ by the thyratron-triggered spark-discharge coupled to a carbon laser plasma with a spark discharge energy of $\sim 0.75 \mathrm{~J}$ and a laser pulse energy of $\sim 50 \mathrm{~mJ} .{ }^{21}$ In these previous experiments, the external electrodes were placed in front of the laser-ablated target. For that configuration, the external electrode distorts the electric field used for ion acceleration. Also, the coupling between the spark discharge and the laser plasma depends on the shape of external electrodes and their distance from the ablated target.

We report on a laser-spark ion source where the carbon target and a grounded mesh serve as the spark's cathode and anode, respectively, resulting in a spark in the direction of the plume expansion. However, this target-mesh electrode configuration can be used only when the target is a conductor. The time delay between the spark-discharge and the laser pulse is varied to optimize the coupling between the spark-discharge and the laser plasma. The ion generation from the laser-spark ion source is compared with the laser ion source with DC electric field in between the target and the mesh. Ion generation of $72 \mathrm{nC}$ with up to $\mathrm{C}^{6+}$ charge state is detected when $1.2 \mathrm{~J}$ spark-discharge energy is coupled to a $\sim 50 \mathrm{~mJ}$ laser pulse. Coupling of the spark energy to the laser plasma offers a compact and cost-effective method to increase the ion charge and yield from the laser plasma generated by small lasers. Spark energy coupling to the laser plasma increases the ion charge and yield without limiting the ion pulse repetition rate, which remains that of the laser.

\section{EXPERIMENTAL}

A schematic of the laser-spark ion source is shown in Fig. 1. A Q-switched Nd:YAG laser with a pulse width of $7 \mathrm{~ns}$, pulse energy of $\leq 80 \mathrm{~mJ}$, and pulse intensity of $\leq 4.5 \times 10^{9} \mathrm{~W} / \mathrm{cm}^{2}$ is used to ablate a $99.99 \%$ pure glassy carbon target $(0.5 \mathrm{~mm}$ thick, $2.5 \mathrm{~cm}$ diameter), mounted on a $5 \mathrm{~cm}$ diameter target holder connected to a vacuum $\mathrm{x}-\mathrm{y}$ manipulator. A grounded nickel mesh of $10 \mathrm{~cm}$ diameter is mounted parallel to the target surface. The base vacuum pressure is maintained in the high $10^{-9}$ Torr. The pressure in the vacuum system increases up to low $10^{-8}$ Torr range during laser ablation. A biconvex lens $\mathrm{L} 1(f=40 \mathrm{~cm})$ is used to focus the laser beam on the target, while the laser beam is incident at $\theta=45^{\circ}$ with the target surface. The laser spot on the target has an area of $\sim 2.5 \times 10^{-3} \mathrm{~cm}^{2}$, as determined by measuring the laser beam width at a target-equivalent-plane with the knife-edge method. A DC voltage of $0-7 \mathrm{kV}$ is applied to the carbon target using a high-voltage power supply (CPS, Inc. $0-30 \mathrm{kV}, 1 \mathrm{~mA}$ ). A pulse forming network (PFN) is constructed to generate a $0-7 \mathrm{kV}$ voltage pulse for the spark-discharge. The PFN consists of 30 high-voltage capacitors (UHV $9 \mathrm{~A}, 2 \mathrm{nF}, 40 \mathrm{kV}$ TKD) mounted on an Al plate and connected with a $1.8-\mathrm{mm}$ thick $\mathrm{Cu}$ wire.

The PFN charging power supply (Glassman, PSLG30R5, $0-20 \mathrm{kV})$ is connected to a current limiting resistor, $R 1=350 \mathrm{k} \Omega$.

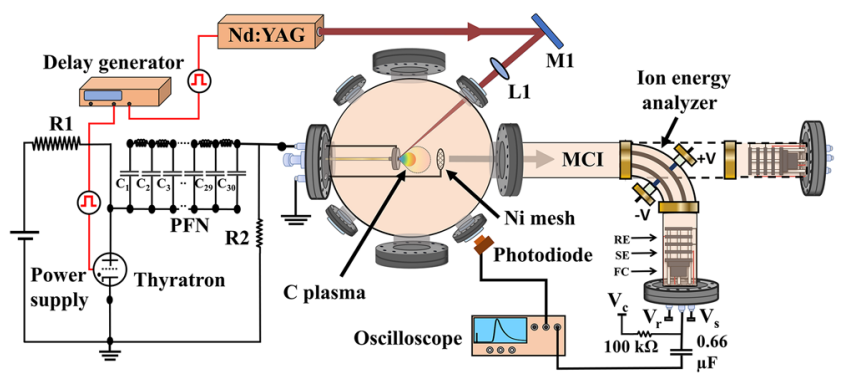

FIG. 1. Laser-spark ion source. The pulse forming network (PFN) is triggered by a delay generator at different time delays with respect to the laser pulse. The spark-discharge is coupled to the carbon plasma as the plasma expands between the carbon target and the Ni mesh. The ion detector consists of a Faraday cup $(\mathrm{FC})$, a secondary electron suppressor ring (SE), and a three-mesh retarding field ion energy analyzer (RE). The straight ion drift configuration is used to detect the total number of ion charges delivered to FC, whereas an electrostatic-ion-energyanalyzer (EIA) is used to select a certain range of ion energy-to-charge ratio. A digital camera is used to capture the image of the plasma. 
The PFN is terminated with a $300 \Omega$ load resistor $R 2$ which is connected in parallel to the spark gap. A digital delay generator (SRSDG 645) is used to externally trigger the thyratron (L-4945A) and the laser pulse. A digital single-lens reflex camera (DSLR-Canon Rebel T3 EOS 1100D, 12.2-megapixel, $0.25 \mathrm{~ms}$ max shutter speed) is used to capture the picture of the plasma plume from an angle of $45^{\circ}$ to the plume expansion direction. The camera is also triggered by the delay generator. The voltage on and current through the target are measured by using a high-voltage probe (Tektronix P6015A, rise time $4.5 \mathrm{~ns}$ ) and a current pick-up coil (Pearson 6595, rise time $2.5 \mathrm{~ns}$ ), respectively. A Si photodiode (Thorlabs DET10A, spectral range $200-1100 \mathrm{~nm}$, rise time $1 \mathrm{~ns}$, active area $0.8 \mathrm{~mm}^{2}$ ) is used to trigger the oscilloscope. Ions with a distribution of energy-tocharge $E / z$ ratio are selected by using an electrostatic ion energy analyzer (EIA) with a radial cylindrical design at a deflection angle of $90^{\circ}$. The range of $E / z$ is obtained by the equation $\frac{E}{z}=\frac{e U}{2 \ln \left(\frac{r_{2}}{r_{1}}\right)}$, where $E$ is the kinetic energy of the ion, $e$ is the electron charge, $U$ is the total voltage across the plates, $r_{1}=14.5 \mathrm{~cm}$ is the inner radius, and $r_{2}=18.2 \mathrm{~cm}$ is the outer radius. The length and inner diameter of the ion transport line are $154 \mathrm{~cm}$ and $10 \mathrm{~cm}$, respectively. The ions are detected at the end of the ion transport line by using a Faraday cup (FC) mounted with a suppressor ring electrode (SE) which is used to suppress the effect of secondary electron emission from ion bombardment of the FC. A three-mesh retarding field ion analyzer (RE) is placed before the SE. The SE and FC are biased at -120 and $-80 \mathrm{~V}$, respectively. The RE is used to measure the ion energy distribution. The two-outer mesh of RE are grounded, while the central mesh is connected to a variable voltage supply $V_{r}$. An oscilloscope (Tektronix DPO 3034) is used to record the time-of-flight (TOF) of the carbon ions through a $0.66 \mu \mathrm{F}$ coupling capacitor. The data are averaged for 30 laser pulses to average-out pulse-to-pulse fluctuations in TOF. A new target surface is exposed to the laser spot after 30 consecutive pulses in order to avoid the formation of deep craters. For a new surface spot, the first few laser pulses are disregarded to remove any possible contamination on the target surface. We noted that when the laser pulses repeatedly hit the same target spot, the total number of detected ions is decreased by $\sim 6 \%$ at the 100th laser pulse compared to the first laser pulse at $3 \times 10^{9} \mathrm{~W} / \mathrm{cm}^{2}$ pulse intensity. The laser intensity was measured before the chamber viewport, and the intensity on the target surface was estimated considering $10 \%$ energy loss mainly due to reflections from the viewport air and vacuum interfaces.

\section{RESULT AND DISCUSSION}

\section{A. Voltage and current}

The ions emitted from the laser plasma are accelerated by the electric field that is applied between the target and the mesh. The voltages on the target for different laser intensities are shown in Fig. 2(a). A $\sim 90 \%$ drop in the voltage is observed after $\sim 4$ and $\sim 2 \mu \mathrm{s}$ at a laser intensity of $3.0 \times 10^{9}$ and $4.5 \times 10^{9} \mathrm{~W} / \mathrm{cm}^{2}$, respectively. The voltage drop occurs when the plasma plume connects the target to the mesh allowing the spark to occur. The current through the target is shown in Fig. 2(b). The maximum current through the target is $1.48 \mathrm{~A}$ for $5 \mathrm{kV}$ target voltage and $0.3 \mathrm{~A}$ for $1 \mathrm{kV}$ target voltage

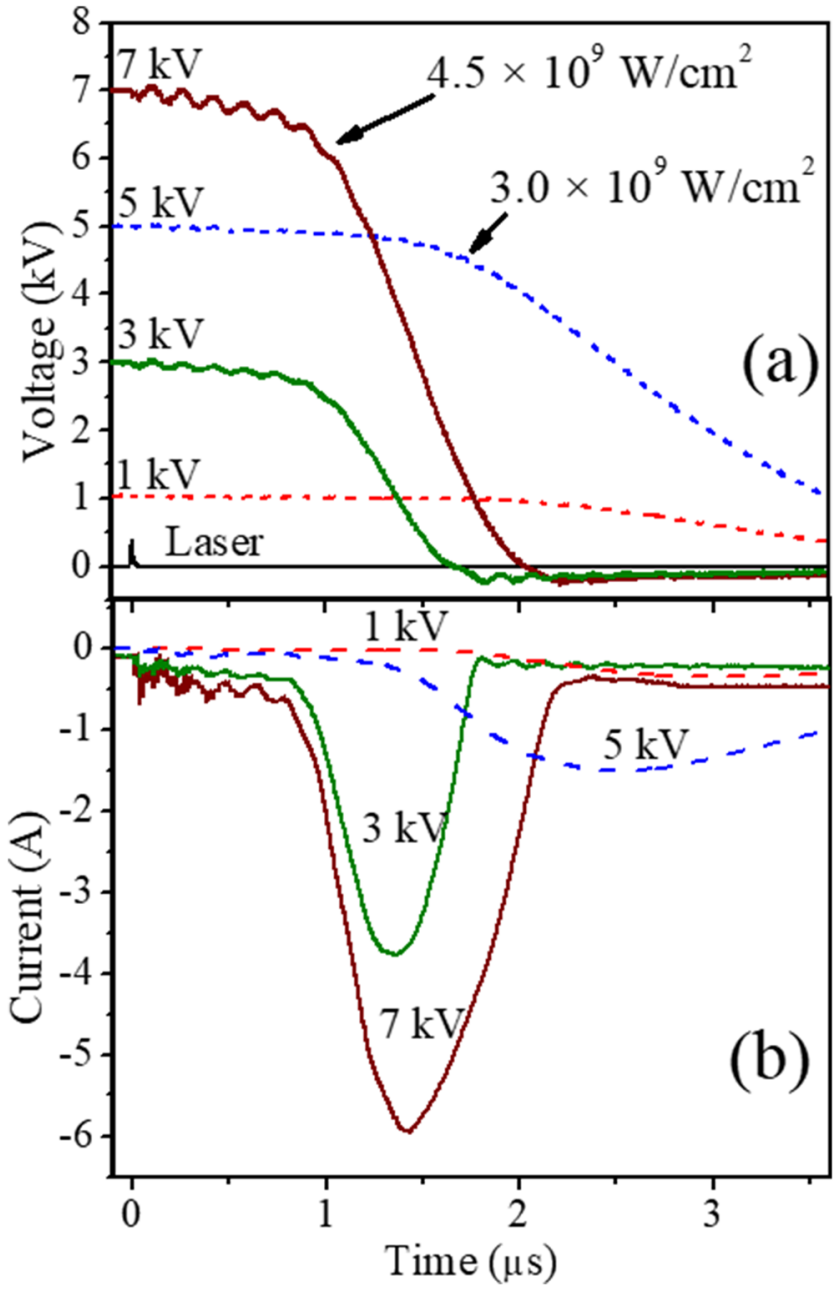

FIG. 2. (a) Voltage on the target and (b) current through the target when the target voltage is varied from 1 to $7 \mathrm{kV}$. The photodiode signal of the laser pulse indicates the laser triggering. The laser pulse intensities are set as $3.0 \times 10^{9}$ (dash line) and $4.5 \times 10^{9} \mathrm{~W} / \mathrm{cm}^{2}$ (solid line), respectively, in both [(a) and (b)].

at a laser intensity of $I=3.0 \times 10^{9} \mathrm{~W} / \mathrm{cm}^{2}$. The maximum current increases up to 6 A for $7 \mathrm{kV}$ target voltage for $I=4.5 \times 10^{9} \mathrm{~W} / \mathrm{cm}^{2}$.

The spark-discharge voltage and current are measured for different time delays $t_{d}$ between the thyratron and the Q-switch trigger pulse provided by a delay generator (Fig. 3). The length of each BNC cable is kept less than $3 \mathrm{~m}$ to avoid delay on rise time. The signal rise times of the photodiode, voltage probe, and current probe are in the order of few ns, as provided by the manufacturer, which is adequate for our experiment. The time delay $t_{d}$ is varied from 0 to $1.6 \mu \mathrm{s}$ for a laser intensity of $3.0 \times 10^{9} \mathrm{~W} / \mathrm{cm}^{2}$. For $t_{d}<0.8 \mu \mathrm{s}$, the spark-discharge is not ignited. A significant rise in current across the target is recorded for $0.8 \mu \mathrm{s}<t_{d}<1.2 \mu \mathrm{s}$, which results from the spark-discharge between the target and the mesh. Over that range of $t_{d}$, the peak spark current decreases gradually with time delay. At $t_{d}>1.6 \mu \mathrm{s}$, the voltage drop across $R 2$ is similar to that for $t_{d}<0.8 \mu \mathrm{s}$, which indicates the absence of the spark between the target and the 


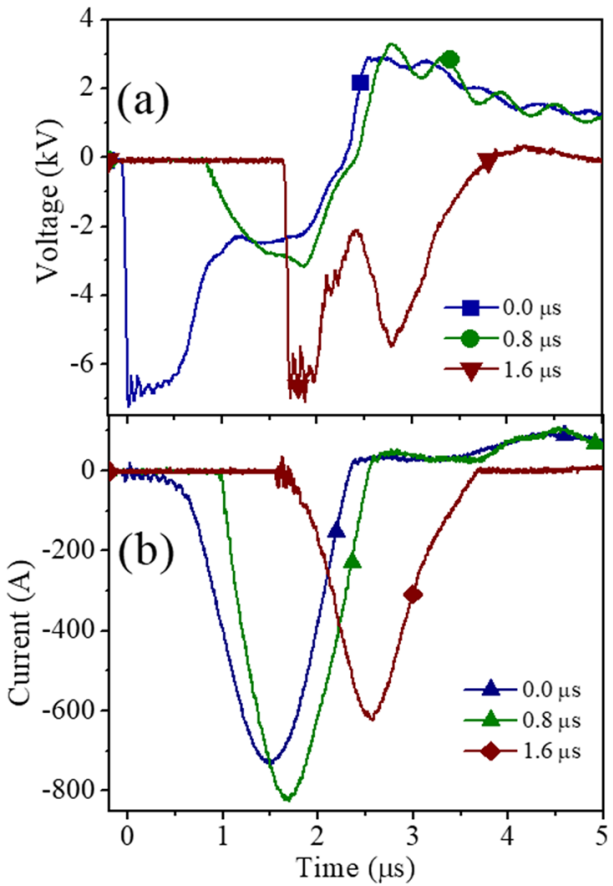

FIG. 3. (a) Voltage on and (b) current through the target. The grounded mesh is placed $10 \mathrm{~cm}$ in front of the target. The time delay between the laser pulse and the PFN trigger is varied from 0 to $1.6 \mu \mathrm{s}$ for $7 \mathrm{kV}$ PFN charging voltage at $3.0 \times 10^{9} \mathrm{~W} / \mathrm{cm}^{2}$ laser intensity.

mesh through the plasma plume. For $I<1.0 \times 10^{9} \mathrm{~W} / \mathrm{cm}^{2}$, the spark-discharge did not occur for all $t_{d}$ as the laser plasma is not dense enough to initiate the spark-discharge.

\section{B. Ion time-of-flight}

The ion velocity generated from a laser plasma has three main components: plume ablation velocity, ion thermal velocity, and ion velocity gain by the double-layer potential at the plume-vacuum interface. ${ }^{35}$ The double-layer potential is established due to the emission of fast electrons, at the early stages of plume expansion, leaving a space-charge region in the front of the expanding plume. The energy that the ions gain in the double-layer potential is proportional to their charge state. The ions can be further accelerated by an external electric field. We refer to the ions that are accelerated by the external electric field as fast ions $q_{\text {fast }}$, while the ions that are not accelerated by the external electric field are referred to as slow ions $q_{\text {slow }}$. Both fast and slow ions are accelerated by the double-layer potential. Fast ion peaks are detected early in the TOF signal. For our target-mesh configuration and laser fluence of $3.0 \times 10^{9} \mathrm{~W} / \mathrm{cm}^{2}$ with the laser plasma coupled to $1.2 \mathrm{~J}$ spark-discharge energy, only $\sim 10 \%$ of the total detected ions are fast ions. Figure 4 shows the TOF of slow ions for a spark-discharge energy of $0-1.2 \mathrm{~J}$ coupled to the laser plasma, which corresponds to charging the PFN to $0-7 \mathrm{kV}$. The total ion charge $Q=\int I(t) d t$, where $I(t)$ is the ion current measured from the FC. The TOF shows that the number of slow ions increases from $\sim 12$ to $\sim 72 \mathrm{nC}$ when the energy of the spark-discharge is gradually

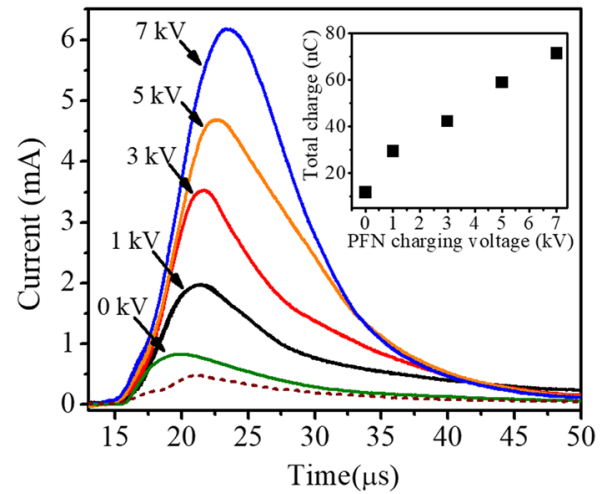

FIG. 4. Time-of-flight of slow ions $\left(q_{\text {slow }}\right)$ for different PFN charging voltages. A delay time of $t_{d}=0.9 \mu \mathrm{s}$ is set between the laser pulse and the spark-discharge. The inset shows that relationship between the total charge and PFN charging voltage. The dotted line is the TOF signal when $3 \mathrm{kVDC}$ accelerating voltage is applied to the target. The laser pulse intensity is fixed at $3.0 \times 10^{9} \mathrm{~W} / \mathrm{cm}^{2}$.

increased from 0 to $1.2 \mathrm{~J}$. The ion TOF peak is also shifted from 19 to $25 \mu$ s which indicates the broadening of the ion energy distribution with higher spark-discharge energy. The TOF of the slow ions generated when the DC voltage is applied to the target is also measured. The total charge of the slow ions detected is $\sim 7 \mathrm{nC}$ when $3 \mathrm{kV}$ DC voltage is applied on the target for $3.0 \times 10^{9} \mathrm{~W} / \mathrm{cm}^{2}$ laser intensity.

The total carbon ion charge detected by the FC for $3 \mathrm{kV}$ external electric field applied to the target is significantly smaller than the total carbon ion charge detected without voltage applied to the target, as shown in Fig. 4 (dotted line). The result can be explained by the divergence of the ions emitted by the laser plasma as a result of the distortion of the extraction field due to the presence of the expanding plasma in the target-mesh region. This field distortion causes the ion beam to diverge strongly before moving into the drift tube, which is intensified by the thermal kinetic energy of the ions whose velocity vectors are not parallel to the drift tube. $^{3}$

The three-mesh retarding field analyzer is used to observe the ion energy distribution. The retarding voltage blocks ions with kinetic energy less than $z e V_{0}$, where $z$ is the ion charge state, $V_{0}$ is the retarding voltage applied to the central electrode of the energy analyzer, and $e$ is the unit charge in Coulomb. The ions with higher energy than $z e V_{0}$ lose some of their energy as they drift toward the retardation mesh; however, they are accelerated back to their initial energy between the central and final mesh of the three-mesh retarding field ion analyzer before being detected by the FC. The retardation of slow ions generated by $\sim 0.6 \mathrm{~J}$ spark-discharge energy coupled to the plasma generated by $3.0 \times 10^{9} \mathrm{~W} / \mathrm{cm}^{2}$ laser pulse is shown in Fig. 5(a). The inset of Fig. 5(a) shows that the number of ions decreases from 45 to $2 \mathrm{nC}$ when the retarding voltage increases from 0 to $250 \mathrm{~V}$. Figure 5(b) shows the retardation of the carbon ions generated by the laser plasma alone with $I=3.0 \times 10^{9} \mathrm{~W} / \mathrm{cm}^{2}$. A retarding voltage of $\sim 250 \mathrm{~V}$ is enough to retard more than $95 \%$ of the ions. The results show that the spark-discharge increases the ion-yield, as well as ion energy, compared to the slow ions generated for the laser pulse only ( $0 \mathrm{kV}$ on target). 


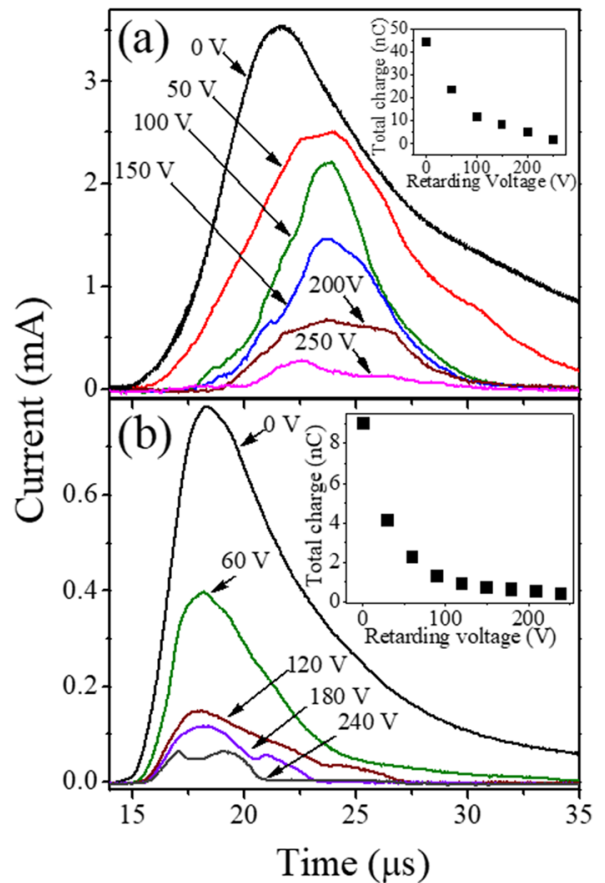

FIG. 5. (a) The retardation of $q_{\text {slow }}$, generated by $\sim 0.6 \mathrm{~J}$ spark-discharge coupled to laser plasma ( $3 \mathrm{kV}$ PFN charging voltage). The inset shows the total number of $q_{\text {slow }}$ with respect to retarding voltage. (b) The retardation of $q_{\text {slow }}$, generated by laser plasma only ( $0 \mathrm{kV}$ on the target). The inset shows a total number of ions at different retarding voltages. For both [(a) and (b)], the laser intensity is $3.0 \times 10^{9} \mathrm{~W} / \mathrm{cm}^{2}$.

The ion generation from a laser-spark source starts by the laser pulse ablating the target to produce laser plasma. Then, the ablated plume expands connecting the target to the grid region, while the spark is triggered to enhance the plasma temperature and density, producing more MCIs. The cathode spots on the target created by the spark-discharge can also act as an ion source. During the spark-discharge, the spark current is concentrated at a small number of cathode spots. The spot is formed by an explosive emission process. The lifetime of a cathode spot can be in the range of $10 \mathrm{~ns}-1 \mu \mathrm{s}$ as previously reported. ${ }^{37,38}$ The plasma pressure within a cathode spot is high, and the strong pressure gradient causes the plasma to move forward from the target surface in a similar way to the plasma plume generated by the focused laser beam at a solid surface. As a result, ions are generated with higher ion energy and temperature for the laser-spark compared to the laser pulse alone. Figure 5(a) shows a broader ion energy distribution and higher ion yield for the spark-discharge compared to Fig. 5(b) which shows ions generated by the laser pulse alone. The spark discharge causes an increase in the plasma temperature and density.

An external DC voltage $V_{\text {ext }}$ is applied between the target and the mesh to accelerate the ions. This electric field produces substantially more kinetic energy to the fast component of the ions $q_{\text {fast }}$ than their thermal kinetic energy $q_{\text {slow }}$. The time-of-flight of $q_{\text {fast }}$ in the drift tube is mainly due to electric field acceleration, which is charge-dependent. The ion-acceleration depends on the temporal and spatial distribution of ions with different charge states. Multicharged ions are generated at the early plasma plume development stage and accelerated to the front of the plume with the higher charges experiencing more acceleration. ${ }^{39}$ As the ions are generated in the gap between the target and the grounded mesh, the ions gain less kinetic energy than the potential drop between the target and the mesh. The Debye shielding of the plume also limits the efficiency of ion extraction, and therefore, the ions acquire final kinetic energy lower than $Z_{i} e V_{e x t}$, where $Z_{i}$ is the ion charge and $V_{e x t}$ is the external voltage applied to the target. The fast ions are accelerated in the electric field, and the retrograde motion of the plasma edge exposes more ions to the external electric field and repels electrons. ${ }^{40,41}$ The total number of ions reaching the FC is calculated from the TOF signal for a straight drift tube, whereas the EIA is used for selecting ions with a range of energy-to-charge $E / z$ ratio. The ion yield of the $q_{\text {fast }}$ is increased by a factor of $\sim 4$ when the target voltage is increased from 3 to $7 \mathrm{kV}$, as shown in Fig. 6(a). The charge states of the fast ions are identified with the help of the EIA since they are well-separated in time, as shown in Fig. 6(b). A slit with a narrow opening at the entrance and exit of EIA can provide higher resolution in $E / z$ selection. However, the EIA is operated without a slit in order to maximize the ion transmission. The resolution of EIA $(\Delta E / E)$ is calculated as $\sim 10 \%$. Figure $6(\mathrm{~b})$ shows the TOF signal detected for different $E / z$ ratios as selected by the voltage applied to the EIA. The value of $E / z$ is set to maximize the ion signal for each accelerating voltage on the target. The maximum ion yield is obtained for $E / z$ set at $1.8,3.2$, and $4.3 \mathrm{keV}$ for DC voltages of 3,5, and $7 \mathrm{kV}$ applied to the target. $\mathrm{Up}$ to $\mathrm{C}^{3+}$ is detected for $3 \mathrm{kV}$ ion accelerating voltage, whereas a $\mathrm{C}^{4+}$ peak is detected for $7 \mathrm{kV} \mathrm{DC}$ voltage on the target. The TOF signal is related to the ion charge $Z_{i}$ by the equation TOF $=\sqrt{\frac{2 m}{Z_{i} e V}} d_{1}+\sqrt{\frac{m}{2 Z_{i} e V}} d_{2}$, where the first term is the time that an ion spends in the gap between the target and the grounded mesh, while the second term is the ion drift time from the mesh to the Faraday cup, $d_{1}$ is the distance from the target to the mesh, $d_{2}$ is the distance from the extraction mesh to the Faraday cup, $m$ is the mass of the carbon atom, $e$ is the electron charge, $Z_{i}$ is the charge state, and $V$ is the effective potential which is the sum of the double-layer potential and the potential that the ions experience in the target-mesh gap. Ions generated in the plasma plume are accelerated to an energy that is about proportional to their charge $Z_{i}{ }^{17,18,20,21}$ However, a subset of ions with low charge states have energies that are slower than that acquired by $V$ and are referred to as slow ions. ${ }^{21}$ These ions are generated by multiphoton and collisional processes. Experimentally, we identified the MCIs using the ratio of calculated TOF of individual MCIs. With the passage of the ions through the EIA, a subset of charges with a narrow range of $E / z$ is selected for each charge state. This gives well-identified peaks that can be easily correlated with the charge state based on their TOF. TOF signals taken using the straight tube, which was setup to have the same ion TOF as when the EIA is in the ion path, show additional peaks, mainly due to the spark voltage structure that affects ion acceleration. Other mechanisms of slow ion generation also affect the TOF signal observed without the EIA. Therefore, the identification of ion charge was based on maximizing ion transmission through the EIA by varying the selected $E / z$ and then correlating the ion signal with the TOF equation. 


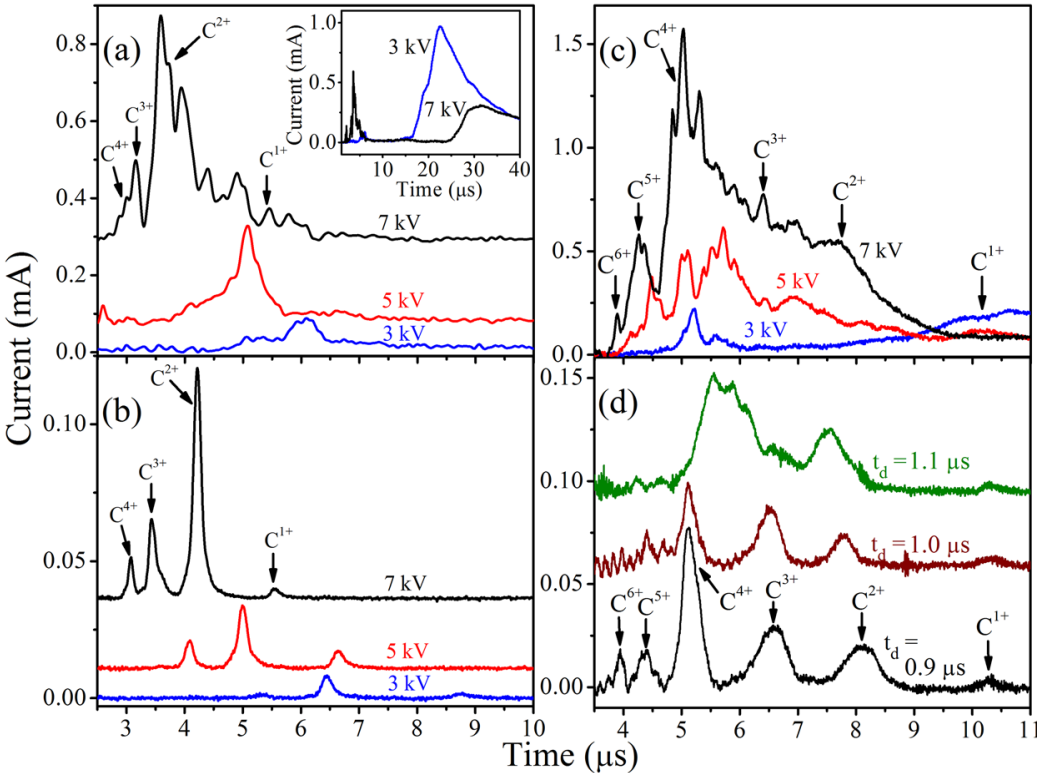

FIG. 6. (a) Time-of-flight signal (TOF) of fast carbon ions detected using the straight drift tube. Up to $\mathrm{C}^{4+}$ is generated for $7 \mathrm{kV}$ DC voltage applied to the target. The inset shows the TOF of slow and fast ions for 7 and $3 \mathrm{kV}$ on target. (b) lons with different charge states transmitted through the electrostatic ion energy analyzer. The energyto-charge selection E/z is adjusted accordingly to maximize the ion current, $E / z=1.8,3.2$, and $4.3 \mathrm{keV}$ for target voltages of 3,5 , and $7 \mathrm{kV}$, respectively. (c) The TOF of the accelerated fast carbon ions for 3,5, and $7 \mathrm{kV}$ PFN charging voltage. (d) The ion TOF signal for different time delays $t_{d}$ between the laser pulse and the spark-discharge. The spark-discharge energy is $1.2 \mathrm{~J}$, and the $E / z$ ratio is $1.3 \mathrm{keV}$. For (a)-(d), the laser pulse intensity is fixed at $I=3.0 \times 10^{9} \mathrm{~W} / \mathrm{cm}^{2}$. (a) and (c) are recorded using a straight drift tube, whereas (b) and (d) are recorded with an EIA analyzer inserted in the ion beam path.
A significant increase in ion detection and charge states is observed for the laser-spark configuration, as shown in Fig. 6(c). The number of fast ions increases from 0.12 to $3.5 \mathrm{nC}$, when the sparkdischarge energy is increased from 0.6 to $1.2 \mathrm{~J}$ (3-7 kV on the PFN). In Fig. 6(d), the ion TOF signal is shown for the $E / z$ ratio centered at $1.3 \mathrm{keV} . \mathrm{C}^{5+}$ and $\mathrm{C}^{6+}$ ions are detected when $1.2 \mathrm{~J}$ spark-discharge energy is coupled to the laser plasma for a laser intensity $I=3.0$ $\times 10^{9} \mathrm{~W} / \mathrm{cm}^{2}$. The time delay $t_{d}$ between the laser pulse and the spark-discharge is varied. A consistent spark-discharge is observed at $t_{d}>0.8 \mu \mathrm{s}$, and up to $\mathrm{C}^{6+}$ is recorded at $t_{d}=0.9 \mu \mathrm{s}$. $\mathrm{C}^{6+}$ and $\mathrm{C}^{5+}$ are not identified for $t_{d}>0.9 \mu$ s as the higher charge state ions are in the front of the plume and pass the mesh without amplification. The ionization energy of the $\mathrm{C}^{1+}, \mathrm{C}^{2+}, \mathrm{C}^{3+}, \mathrm{C}^{4+}, \mathrm{C}^{5+}$, and $\mathrm{C}^{6+}$ ions is $11.26,24.38,47.89,64.49,392.09$, and $489.99 \mathrm{eV}$, respectively. ${ }^{42}$ The yield of each ion charge is dependent on the rate of ionization collisions. Higher plasma temperatures at the core of the laser plume, where most MCIs are generated, result in collisional ionization of the lower ion charges to higher ones. The strong $\mathrm{C}^{4+}$ peak results from collisional ionization of the lower charge ions, which is also evident from the weak $\mathrm{C}^{1+}$ peak. Further ionization beyond $\mathrm{C}^{4+}$ to form $\mathrm{C}^{5+}$ and $\mathrm{C}^{6+}$ is limited by the required ionization energies. Previous results on spark and laser $\mathrm{C}$ ion sources have also shown ion TOF signals that peak for $\mathrm{C}^{4+}$, depending on the energy deposited in the plasma. ${ }^{33,4}$

The cross sections for the interaction of different MCIs, ranging from $\mathrm{H}^{+}$to $\mathrm{Ca}^{20+}$, with hydrogen gas were previously measured. Considering an MCI with $100 \mathrm{eV} /$ unit charge, the mean free path of the MCI is orders of magnitude longer than our experimental drift tube length. Therefore, the ion charge remains almost unchanged in the drift tube.

A digital camera is used to record the enhancement of optical emission from the laser-spark plasma plume from that with the laser alone. The camera is triggered by the delay generator at a fixed delay of $1.5 \mathrm{~ms}$ from the laser pulse. The plume size increases when the DC and pulsed voltage is applied to the target. The time delay $t_{d}$ is fixed at $0.9 \mu$ s between the laser pulse and spark-discharge. Figure 7 (a) shows a bright plasma core at the point of interaction of the laser beam and the target surface. The plasma core length is $\sim 0.5 \mathrm{~cm}$ for $4.5 \times 10^{9} \mathrm{~W} / \mathrm{cm}^{2}$ laser intensity. Figure $7(\mathrm{~b})$ shows that the plasma core is extended up to $\sim 1 \mathrm{~cm}$ when $7 \mathrm{kV}$ DC voltage is applied to the target. Further enhancement of the carbon plasma plume is observed in Fig. 7(c) when a pulsed voltage is applied to the target. We also noticed the generation of macroparticles from the carbon plasma when spark discharge is initiated. Carbon macroparticles

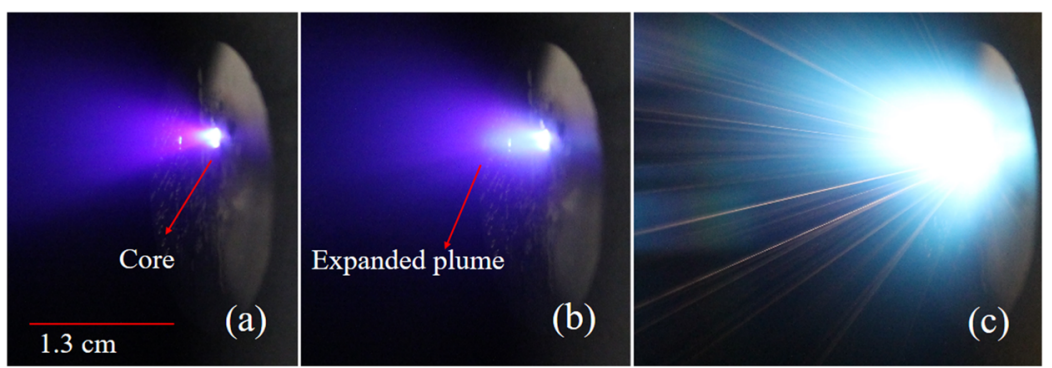

FIG. 7. (a) The image of carbon plasma captured by using a digital camera for laser pulse alone, (b) for $7 \mathrm{kV}$ DC voltage on target, and (c) for $1.2 \mathrm{~J}$ spark-discharge energy coupled to a laser plasma. Laser pulse intensity is fixed at $3.0 \times 10^{9}$ $\mathrm{W} / \mathrm{cm}^{2}$. 
are incandescently glowing species that were previously reported for carbon target ablation by a laser intensity of $5 \times 10^{10} \mathrm{~W} / \mathrm{cm}^{2}{ }^{45}$ The generation of macroparticles is also well known in vacuumarc deposition. ${ }^{46}$ Some macroparticles appear much brighter than others. The brightness changes due to the different speed of individual macroparticles; a slow macroparticle produces an intense exposure to the camera detector than the fast particle. ${ }^{47}$ The purple colored region in Fig. 7 indicates the presence of low temperature plasma, whereas the bright color indicates the presence of hot plasma. The plasma plume images are captured for long integration times resulting in the observation of traces of light-emitting carbon macroparticles. The observation time of the traces of the carbon macroparticles was reported at 30-120 $\mu$ s after the onset of plasma formation. ${ }^{4}$

The camera is triggered by applying a reset pulse from the delay generator. The time delay of the camera exposure and sparkdischarge triggering is estimated to be $1.5 \mathrm{~ms} .20$ images are taken for a similar time delay to ensure the repeatability. Similar experimental setup was used in a previous study.

\section{CONCLUSION}

Carbon ions with a charge up to $\mathrm{C}^{6+}$ are generated by $1.2 \mathrm{~J}$ spark-discharge energy coupled to carbon plasma generated by $50 \mathrm{~mJ}$ laser pulse ablation of a C target, whereas only ions with charges up to $\mathrm{C}^{4+}$ are detected with a DC voltage applied to the target for similar conditions. A time delay between the spark-discharge and the laser pulse of $\sim 0.9 \mu$ s maximizes the generated charge and charge state. This geometry of coupling the spark discharge energy to the expanding plasma plume with the electric field along the direction of plume expansion enhances the total ion generation and reduced the complexity of the laser-spark discharge for ion generation.

\section{ACKNOWLEDGMENTS}

This material is based on the work supported by the National Science Foundation, USA, under Grant No. MRI-1228228.

\section{REFERENCES}

${ }^{1}$ T. Fujihana, A. Sekiguchi, Y. Okabe, K. Takahashi, and M. Iwaki, "Effects of room temperature carbon, nitrogen and oxygen implantation on the surface hardening and corrosion protection of iron," Surf. Coat. Technol. 51, 19-23 (1992).

${ }^{2}$ S. Witanachchi, A. M. Miyawa, and P. Mukherjee, "Highly ionized carbon plasma generation by dual-laser ablation for diamond-like carbon film growth," MRS Proc. 617, J3.6 (2000).

${ }^{3}$ H. Tsujii, T. Kamada, T. Shirai, K. Noda, H. Tsuji, and K. Karasawa, Carbon-Ion Radiotherapy (Springer, Tokyo, Japan, 2014).

${ }^{4}$ G. Impellizzeri, V. Scuderi, L. Romano, E. Napolitani, R. Sanz, R. Carles, and V. Privitera, "C ion-implanted $\mathrm{TiO}_{2}$ thin film for photocatalytic applications," J. Appl. Phys. 117, 105308 (2015).

${ }^{5}$ J. Kim, G. Lee, and J. Kim, "Wafer-scale synthesis of multi-layer graphene by high-temperature carbon ion implantation," Appl. Phys. Lett. 107, 033104 (2015).

${ }^{6}$ J. D. Gillaspy, "Highly charged ions," J. Phys. B: At., Mol. Opt. Phys. 34, R93 (2001).

${ }^{7}$ K. Amemiya, J. Ito, and K. Tokiguchi, "High energy aluminum ion implantation using a variable energy radio frequency quadrupole implanter," J. Vac. Sci. Technol., A 16, 472-476 (1998).

${ }^{8}$ J. D. Gillaspy, D. C. Parks, and L. P. Ratliff, "Masked ion beam lithography with highly charged ions," J. Vac. Sci. Technol., B: Microelectron. Nanometer Struct. 16, 3294-3297 (1998).
${ }^{9}$ F. Aumayr and H. Winter, "Potential sputtering," Philos. Trans. R. Soc., A 362, 77 (2004)

${ }^{10}$ W. Takahashi, M. Nakajima, N. Yamamoto, S. Yamada, H. Yamashita, K. Nakagawa, H. Tsuji, and T. Kamada, "Carbon ion radiotherapy for oligorecurrent lung metastases from colorectal cancer: A feasibility study," Radiat. Oncol. 9, 68 (2014).

${ }^{11}$ T. Ohno, "Particle radiotherapy with carbon ion beams," EPMA J. 4, 9 (2013).

${ }^{12}$ N. J. Peacock and R. S. Pease, "Sources of highly stripped ions," J. Phys. D: Appl. Phys. 2, 1705 (1969).

${ }^{13}$ V. B. Kutner, Y. A. Bykovsky, V. P. Gusev, Y. P. Kozyrev, and V. D. Peklenkov, "The laser ion source of multiply charged ions for the U-200 LNR JINR cyclotron," Rev. Sci. Instrum. 63, 2835-2837 (1992).

${ }^{14}$ D. Hiroyuki, N. Mamiko, and S. P. Alexander, "Review of laser-driven ion sources and their applications," Rep. Prog. Phys. 75, 056401 (2012).

${ }^{15}$ K. Bernhardi, G. Fuchs, M. A. Goldman, H. C. Herbert, D. Obermann, W. Walcher, and K. Wiesemann, "Studies of electron heating and multiply charged ion production in an electron cyclotron resonance plasma," Plasma Phys. 18, 77 (1976).

${ }^{16}$ A. L. Morton, R. E. Marrs, J. R. Henderson, D. A. Knapp, and B. S. Marilyn, "The electron beam ion trap: A new instrument for atomic physics measurements," Phys. Scr. T22, 157 (1988).

${ }^{17}$ M. H. A. Shaim and H. E. Elsayed-Ali, "Aluminum multicharged ion generation from laser plasma," Nucl. Instrum. Methods Phys. Res., Sect. B 356, 75-80 (2015).

${ }^{18} \mathrm{O}$. Balki and H. E. Elsayed-Ali, "Multicharged carbon ion generation from laser plasma," Rev. Sci. Instrum. 87, 113304 (2016).

${ }^{19}$ H. Kashiwagi, M. Fukuda, M. Okamura, R. A. Jameson, T. Hattori, N. Hayashizaki, K. Sakakibara, J. Takano, K. Yamamoto, Y. Iwata, and T. Fujimoto, "Acceleration of high current fully stripped carbon ion beam by direct injection scheme," Rev. Sci. Instrum. 77, 03 B305 (2006).

${ }^{20}$ M. H. A. Shaim and H. E. Elsayed-Ali, "Spark discharge coupled laser multicharged ion source," Rev. Sci. Instrum. 86, 073304 (2015).

${ }^{21}$ O. Balki, M. M. Rahman, S. Xiao, and H. E. Elsayed-Ali, "Generation of $\mathrm{C}^{6+}$ in a spark-discharge coupled laser plasma," Opt. Commun. 403, 50-54 (2017).

${ }^{22}$ I. G. Brown, "Vacuum arc ion sources," Rev. Sci. Instrum. 65, 3061-3081 (1994).

${ }^{23}$ M. M. M. Bilek, P. J. Martin, and D. R. McKenzie, "Influence of gas pressure and cathode composition on ion energy distributions in filtered cathodic vacuum arcs," J. Appl. Phys. 83, 2965-2970 (1998).

${ }^{24}$ A. Anders, "A brief history of cathodic arc coating," in Cathodic Arcs: From Fractal Spots to Energetic Condensation, edited by A. Anders (Springer New York, New York, NY, 2008), pp. 1-68.

${ }^{25}$ P. A. Lindfors, W. M. Mularie, and G. K. Wehner, "Cathodic arc deposition technology," Surf. Coat. Technol. 29, 275-290 (1986).

${ }^{26}$ E. Hantzsche, "Theories of cathode spots," in Handbook of Vacuum Arc Science and Technology, edited by R. L. Boxman, D. M. Sanders, and P. J. Martin (William Andrew Publishing, Park Ridge, NJ, 1996), pp. 151-208.

${ }^{27}$ J. Kutzner and H. C. Miller, "Integrated ion flux emitted from the cathode spot region of a diffuse vacuum arc," J. Phys. D: Appl. Phys. 25, 686 (1992).

${ }^{28}$ H. J. Scheibe, P. Siemroth, W. Pompe, and B. Schoeneich, "Laser-arc: A new method for preparation of diamond-like carbon films," Surf. Coat. Technol. 47, 455-464 (1991)

${ }^{29}$ H. J. Scheibe, B. Schultrich, R. Wilberg, and M. Falz, "Laser-Arc technology for industrial hard coating deposition," Surf. Coat. Technol. 97, 410-413 (1997).

${ }^{30}$ A. B. Girum, T. Isaac, J. Larissa, H. Patrick, O. S. Gerry, S. Emma, S. Z. Vassily, V. Z. Sergey, and O. R. Fergal, "Laser-assisted vacuum arc extreme ultraviolet source: A comparison of picosecond and nanosecond laser triggering," J. Phys. D: Appl. Phys. 49, 225201 (2016).

${ }^{31}$ J. S. Lash, R. M. Gilgenbach, and H. L. Spindler, "Characterization of a laser-ablation-assisted-plasma-discharge-metallic ion source," Plasma Sources Sci. Technol. 4, 511 (1995).

${ }^{32}$ A. Henig, S. Steinke, M. Schnürer, T. Sokollik, R. Hörlein, D. Kiefer, D. Jung, J. Schreiber, B. M. Hegelich, X. Q. Yan, J. Meyer-ter-Vehn, T. Tajima, P. V. Nickles, W. Sandner, and D. Habs, "Radiation-pressure acceleration of ion beams driven by circularly polarized laser pulses," Phys. Rev. Lett. 103, 245003 (2009). 
${ }^{33}$ S. Takagi, S. Ohtani, K. Kadota, and J. Fujita, "Collision experiment on highly ionized ions using a vacuum spark source," Nucl. Instrum. Methods Phys. Res. 213, 539-544 (1983)

${ }^{34}$ M. M. Rahman, O. Balki, and H. E. Elsayed-Ali, "Diagnostics of a sparkdischarge coupled to laser aluminum plasma by optical emission spectroscopy and ion time-of-flight," Opt. Laser Technol. 111, 762-769 (2019).

${ }^{35}$ N. M. Bulgakova, A. V. Bulgakov, and O. F. Bobrenok, "Double layer effects in laser-ablation plasma plumes," Phys. Rev. E 62, 5624-5635 (2000).

${ }^{36}$ P. Yeates, J. T. Costello, and E. T. Kennedy, "The DCU laser ion source," Rev. Sci. Instrum. 81, 043305 (2010).

${ }^{37}$ A. Anders, S. Anders, B. Juttner, W. Botticher, H. Luck, and G. Schroder, "Pulsed dye laser diagnostics of vacuum arc cathode spots," IEEE Trans. Plasma Sci. 20, 466-472 (1992).

${ }^{38} \mathrm{P}$. Siemroth, B. Schultrich, and T. Schülke, "Fundamental processes in vacuum arc deposition," Surf. Coat. Technol. 74-75, 92-96 (1995).

${ }^{39}$ Y. Lin, R. Xu, L. Li, W. Hang, J. He, and B. Huang, "Kinetic energy and spatial distribution of ions in high irradiance laser ionization source," J. Anal. At. Spectrom. 26, 1183-1190 (2011).

${ }^{40}$ F. F. Chen, "Decay of a plasma created between negatively biased walls," Phys. Fluids 25, 2385-2387 (1982).
${ }^{41} \mathrm{~K}$. Yamada, T. Tetsuka, and Y. Deguchi, "New scaling relation for ion extraction by external electric field on a barium plasma produced between parallel-plate electrodes," J. Appl. Phys. 69, 6962-6967 (1991).

${ }^{42}$ See http://physics.nist.gov/cgi-bin/ASD/ie.pl for NIST Atomic Spectra Database Ionization Energies Data; accessed 19 August 2019.

${ }^{43}$ O. Balki, M. M. Rahman, and H. E. Elsayed-Ali, "Optical emission spectroscopy of carbon laser plasma ion source," Opt. Commun. 412, 134-140 (2018).

${ }^{44} \mathrm{H}$. Ryufuku and T. Watanabe, "Total and partial cross sections for charge transfer in collisions of multicharged ions with atomic hydrogen," Phys. Rev. A 20, 1828-1837 (1979).

${ }^{45}$ S. S. Harilal, A. Hassanein, and M. Polek, "Late-time particle emission from laser-produced graphite plasma," J. Appl. Phys. 110, 053301 (2011).

${ }^{46} \mathrm{~A}$. Anders, "Approaches to rid cathodic arc plasmas of macro- and nanoparticles: A review," Surf. Coat. Technol. 120-121, 319-330 (1999).

${ }^{47}$ A. Anders, "Interaction of vacuum-arc-generated macroparticles with a liquid surface," Appl. Phys. Lett. 73, 3199-3201 (1998).

${ }^{48} \mathrm{~S}$. Thomas and A. Anders, "Velocity distribution of carbon macroparticles generated by pulsed vacuum arcs," Plasma Sources Sci. Technol. 8, 567-571 (1999). 